ARTICLE

https://doi.org/10.1038/s41467-019-12224-3

\title{
Derailing the aspartate pathway of Mycobacterium tuberculosis to eradicate persistent infection
}

\author{
Erik J. Hasenoehrl ${ }^{1}$, Dannah Rae Sajorda ${ }^{1}$, Linda Berney-Meyer ${ }^{1}$, Samantha Johnsonn ${ }^{1}$ JoAnn M. Tufariello ${ }^{1,6}$,
} Tobias Fuhrer (D) ${ }^{2}$, Gregory M. Cook ${ }^{3,4}$, William R. Jacobs Jr. ${ }^{1,5} \&$ Michael Berney (D) ${ }^{1}$

A major constraint for developing new anti-tuberculosis drugs is the limited number of validated targets that allow eradication of persistent infections. Here, we uncover a vulnerable component of Mycobacterium tuberculosis (Mtb) persistence metabolism, the aspartate pathway. Rapid death of threonine and homoserine auxotrophs points to a distinct susceptibility of $M t b$ to inhibition of this pathway. Combinatorial metabolomic and transcriptomic analysis reveals that inability to produce threonine leads to deregulation of aspartate kinase, causing flux imbalance and lysine and DAP accumulation. Mtb's adaptive response to this metabolic stress involves a relief valve-like mechanism combining lysine export and catabolism via aminoadipate. We present evidence that inhibition of the aspartate pathway at different branch-point enzymes leads to clearance of chronic infections. Together these findings demonstrate that the aspartate pathway in Mtb relies on a combination of metabolic control mechanisms, is required for persistence, and represents a target space for anti-tuberculosis drug development.

\footnotetext{
${ }^{1}$ Department of Microbiology and Immunology, Albert Einstein College of Medicine, Bronx, NY, USA. ${ }^{2}$ Institute of Molecular Systems Biology, Swiss Federal Institute of Technology, Zurich, Switzerland. ${ }^{3}$ Department of Microbiology and Immunology, School of Biomedical Sciences, University of Otago, Dunedin 9054, New Zealand. ${ }^{4}$ Maurice Wilkins Centre for Molecular Biodiscovery, The University of Auckland, Private Bag 92019 , Auckland 1042, New Zealand. ${ }^{5}$ Department of Molecular Genetics, Albert Einstein College of Medicine, Bronx, NY, USA. ${ }^{6}$ Present address: Center for Microbial Pathogenesis, Institute for Biomedical Sciences, Georgia State University, Atlanta, GA 30303, USA. Correspondence and requests for materials should be addressed to M.B. (email: michael.berney@einstein.yu.edu)
} 
M ycobacterium tuberculosis $(M t b)$, the causative agent of tuberculosis (TB), is the deadliest pathogen worldwide, with 1.8 million deaths in 2016 , and is the source of severe societal and economic burdens ${ }^{1}$. Mycobacterium tuberculosis is able to establish a devastating chronic infection in humans, where it can withstand host adaptive immunity and survive in granulomas, a phase commonly referred to as persistence. Many drugs are ineffective during persistence, most likely due to reduced penetrance into the devascularized granuloma environment ${ }^{2}$ and enrichment of phenotypically drug-tolerant cells. As a result, treatment regimens require a lengthy 6-months of combination therapy to clear the infection, which greatly increases the risk of antibiotic resistance acquisition ${ }^{1,3}$.

Recent efforts to target $M t b$ during persistence have focused on inhibiting previously ignored metabolic pathways ${ }^{4,5}$. Several studies have demonstrated that central carbon metabolism (CCM) is required during persistence, and several targets have been identified as essential in both acute and chronic infections in mice ${ }^{4-11}$. Importantly, biosynthetic pathways that utilize TCA cycle intermediates, such as amino acid biosynthesis, are also required for $M t b$ survival $^{12}$. However, none of the amino acid biosynthetic pathways of $M t b$ have yet been shown to be essential during chronic infection and it is largely unknown if such building blocks can be scavenged from the host during persistence.

We recently reported that methionine biosynthesis in $M t b$ is required to establish infections in immunocompetent and immunocompromised mice ${ }^{13}$. Methionine, along with isoleucine, threonine, and lysine make up the family of essential amino acids synthesized from aspartate via the aspartate pathway. This pathway is also involved in the biosynthesis of the peptidoglycan building block, diaminopimelate (DAP), and the cofactor, Sadenosyl methionine (SAM); both of which are required for survival in mycobacteria ${ }^{13,14}$. Because of its branched nature and pivotal role in essential cellular processes (cell wall biosynthesis, translation, and one-carbon metabolism), metabolic regulation of the aspartate pathway is likely important to meet precursor requirements during growth and persistence in the host ${ }^{15}$.

The apparent essentiality of the numerous metabolic products and absence of the aspartate pathway in humans and animals ${ }^{15}$ led us to investigate the effects of inhibition of the aspartate pathway in $M t b$ and its requirement during persistence in the host. We hypothesized that disruption of the aspartate pathway leads to a metabolic imbalance and eventual collapse of this essential biosynthetic network. To investigate this, we employed a combinatorial approach of mycobacterial genetics, transcriptomics, metabolomics, biochemical analysis, and animal experiments. Here, we show that both homoserine auxotrophy $(\Delta t h r A)$ and threonine auxotrophy $(\Delta t h r B)$ are bactericidal, and that methionine and threonine are jointly required for survival and persistence. Transcriptomic and metabolomic studies revealed that threonine starvation leads to an unexpected accumulation of lysine, which the cell responds to by activating lysine export and a mycobacteria-specific degradation pathway. Finally, we demonstrate the absolute requirement of the branch-point enzymes homoserine dehydrogenase (ThrA, Rv1294) and homoserine transacetylase (MetX, formerly known as MetA, Rv3341) in both acute and chronic tuberculosis infections. Taken together our work identifies and characterizes a vulnerable component of $M t b$ 's persistence metabolism with its metabolic control mechanism and puts it on the map for drug discovery.

\section{Results}

Threonine and homoserine auxotrophy are bactericidal. The aspartate pathway produces essential proteinogenic amino acids threonine, methionine, lysine, and isoleucine, the co-factor Sadenosyl methionine, and the cell wall component diaminopimelate. To dissect the individual contributions of pathway branches to metabolic homeostasis and pathogen survival, we attempted to delete selected branch-point enzymes to create auxotrophic strains in $M t b$. These auxotrophic strains could then be used to study metabolic alterations in vitro and serve as metabolic probes in vivo. We have previously shown that MetX (formerly known as MetA), a homoserine transacetylase that converts homoserine to $O$-acetyl-L-homoserine, is essential for $M t b$ survival $^{13}$. Upstream of MetX lies an important branch point of the aspartate pathway because homoserine is needed for both threonine and methionine biosynthesis (Fig. 1a). In order to dissect the effects of combined methionine/threonine and single threonine or methionine auxotrophy, we made null deletion mutants of homoserine dehydrogenase (ThrA) and homoserine kinase (ThrB) in $M t b \mathrm{H} 37 \mathrm{Rv}$, leading to the mutant strains $\Delta t h r A$ and $\Delta t h r B$, respectively. Deletion of $t h r B$ yielded a threonine auxotroph (Fig. 1b), which was killed rapidly in vitro in the absence of threonine supplementation (Fig. 1c). Death in this mutant could not be rescued by addition of isoleucine. Deletion of thrA was bactericidal and could be rescued by either the addition of homoserine or by a combination of methionine and threonine (Fig. 1d). Interestingly, the addition of either methionine or threonine alone in $\Delta t h r A$ led to a statistically significant slowdown of cell killing (Fig. 1e).

Threonine auxotrophy leads to accumulation of lysine and catabolites. We next sought to elucidate the cellular consequences to inhibition at the threonine- homoserine-methionine branchpoint. We employed a combinatorial transcriptomic and metabolomics approach. mRNA transcript levels of the $\Delta t h r B$ and $\Delta t h r A$ mutant strains were measured by RNA-seq and microarray analysis, respectively, in time-course experiments in growth media lacking the amino acids required for survival (Fig. 2). The $\Delta t h r B$ and $\Delta t h r A$ strains have an expression profile similar to that previously observed in methionine starvation (Supplementary Data 2) ${ }^{13}$ and general nutrient starvation (Supplementary Data 2$)^{16}$, as evidenced by the downregulation of lipid biosynthesis and respiration-related genes, and upregulation of pyruvate and succinate metabolism (Fig. 2). However, there were also significant differences in gene expression that indicated killing by threonine and homoserine auxotrophy occurs via a mechanism distinct from that mediated by methionine or general starvation ${ }^{13,16}$. Threonine and homoserine auxotrophy each led to a particularly strong and early (within 2 days) response (upregulation) in redox stress gene expression (Fig. 2). The strongest changes were observed in genes involved in lysine degradation and export pathways. The third-most upregulated gene (8-fold) was the lysine- $\varepsilon$ aminotransferase, lat (Rv3290c) (Fig. 2), which catalyzes degradation of lysine to $\alpha$-aminoadipic acid semialdehyde (Fig. 1a). The lat gene has been identified as a persistence-related gene in $M t b$ and $M$. smegmatis ${ }^{16-18}$. The concurrent upregulation of $p c d$ (Rv3293) (Fig. 2), which further degrades $\alpha$-aminoadipic acid semialdehyde to $\alpha$-aminoadipate, and Rv1986, a probable lysine exporter ${ }^{19}$, suggests a general upregulation in lysine disposal pathways.

To investigate if these substantial transcriptional changes also translate into metabolic changes, we characterized the intracellular and extracellular metabolomes by ultra-performance liquid chromatography-mass spectrometry (UPLC-MS). In accordance with the upregulation of genes for lysine disposal, the $\Delta t h r A$ mutant in media lacking homoserine displayed a significant increase (up to 100-fold) of all identifiable metabolites in the lysine biosynthesis pathway (Fig. 3a). Diaminopimelate, lysine, $\alpha$ aminoadipate, and $\mathrm{N}$-acetylysine were all found in at least 10 -fold 
a
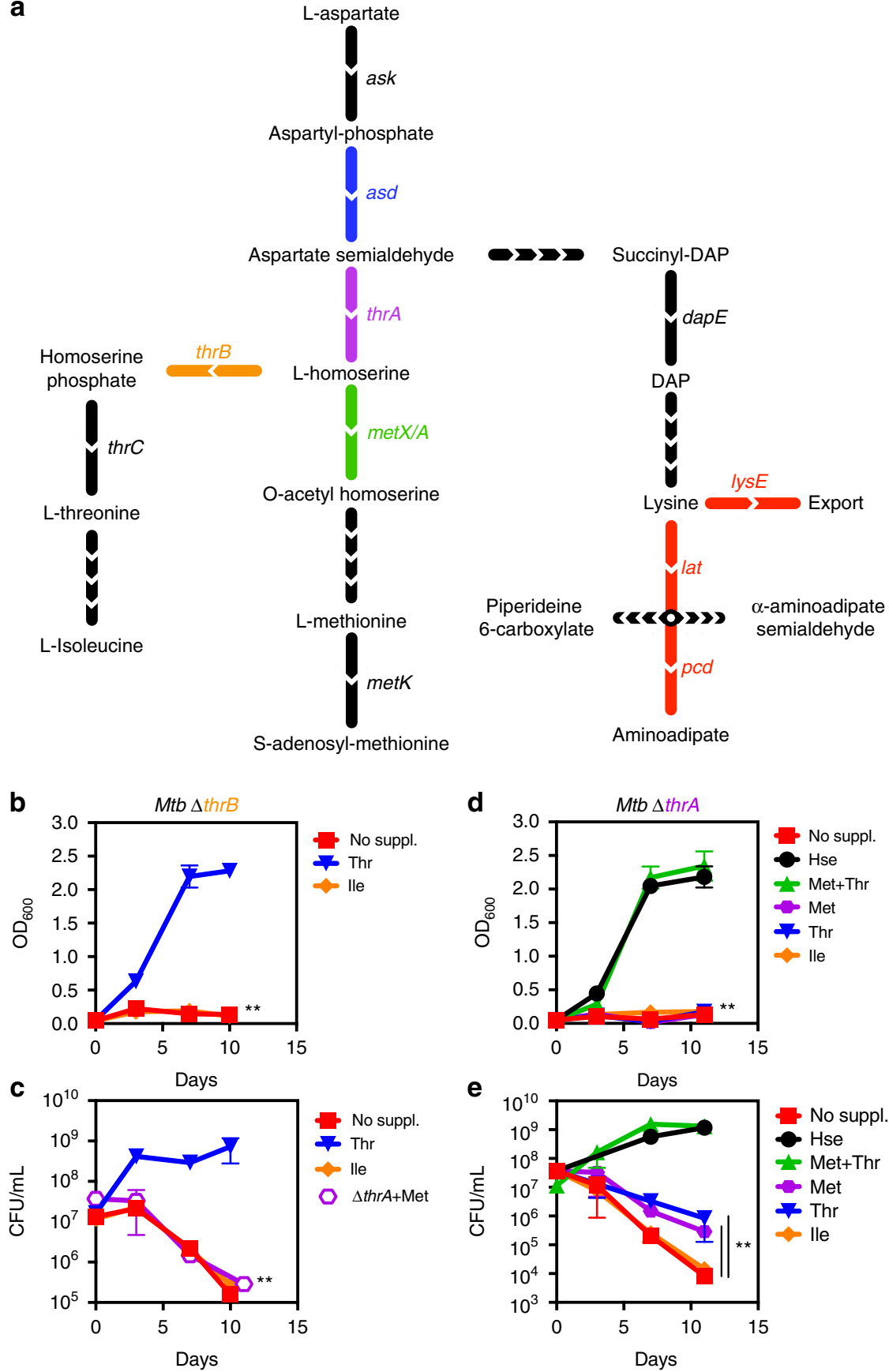

Fig. 1 Inactivation of homoserine dehydrogenase and homoserine kinase is bactericidal. a The aspartate family amino acid biosynthesis pathway (aspartate pathway) in M. tuberculosis including the lysine degradation (aminoadipate) pathway. Ask: aspartate kinase, Asd: aspartate semialdehyde dehydrogenase, ThrA: threonine dehydrogenase, MetX: homoserine transacetylase, ThrB: homoserine kinase, ThrC: threonine synthase, MetK: S-adenosyl methionine synthase, DapE: Succinyl-diaminopimelate desuccinylase, LysE: lysine exporter, Pcd: aminoadipate semialdehyde dehydrogenase, Lat: lysine transacetylase. Two mutants were constructed, one threonine auxotroph Mtb $\Delta t h r B$ and Mtb $\Delta t h r A$, a strain auxotrophic for both, threonine and methionine. $\mathbf{b}$, $\mathbf{d}$ Growth measured by optical density $\mathrm{OD}_{600}$. c, e Survival curves measured by colony forming units (CFU). b Deletion of thrB can be chemically complemented by threonine (Thr) (blue inverted triangles) but not isoleucine (Ile). c Deletion of thrB is bactericidal and mirrors the kill curve of Mtb $\Delta$ thrA supplemented with methionine (Met) (open rhombus). d Deletion of thrA can be chemically complemented by homoserine (Hse) (black circles) or a combination of threonine and methionine (blue triangles), but not by threonine, isoleucine (orange diamonds) and methionine alone (purple rhombus). e Deletion of thrA is bactericidal. Cell death of $M t b \Delta t h r A$ can be slowed by addition of threonine or methionine but not isoleucine, indicating that inhibition of each individual branch (threonine and methionine) contributes to killing. All values are the average of three biological replicates $(n=3) \pm$ s.d. (error bars show standard deviation) and are representative of a minimum of two independent experiments. ${ }^{\star \star} p$-value $<0.01$ in Student's $t$-test. Source data are provided as a Source Data file 
a

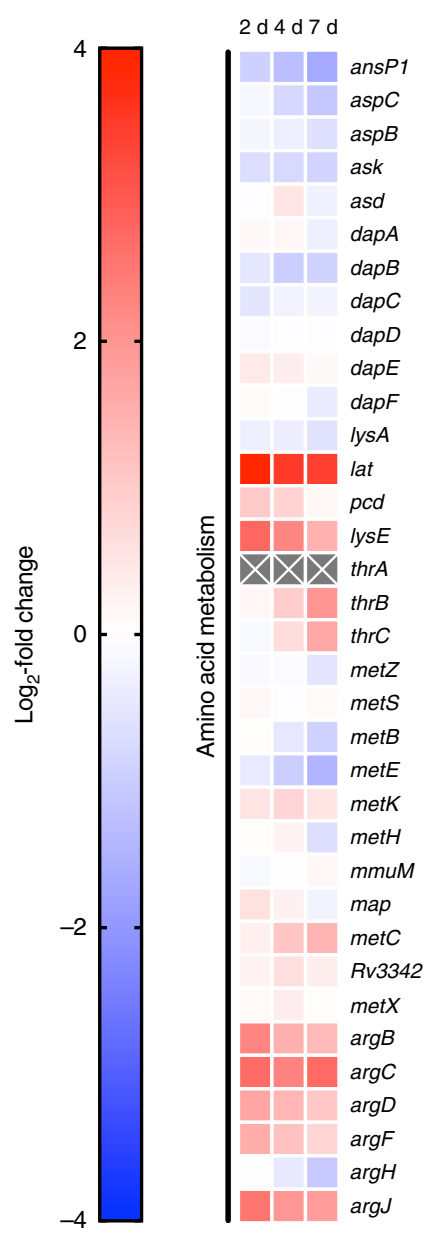

b $\quad \Delta$ thrB

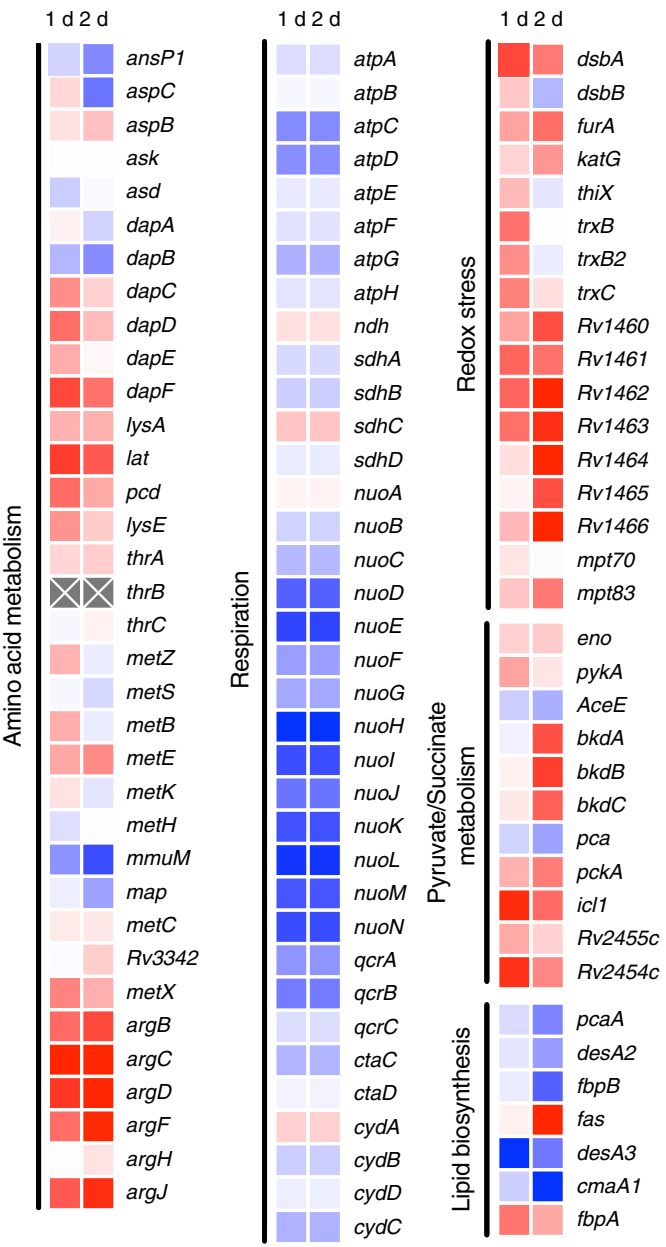

Fig. 2 Time-course transcriptome of $M t b \Delta t h r A$ and $\Delta t h r B$ mutants. Time-course transcriptomic profile of the $\Delta$ thrA (a) and $\Delta$ thrB (b) mutants during homoserine and threonine starvation, respectively. Samples from $n=4(\Delta t h r A)$ and $n=3(\Delta t h r B)$ biologically independent replicates were harvested on days $0,2,4$, and 7 for $\Delta$ thrA and days 0,1 , and 2 for $\Delta$ thrB, and RNA was extracted. Scale is represented in log 2 -fold change compared to day 0 in supplemented media. Genes in relevant pathways are presented. Gene expression ratios were considered statistically significant if there was a $p$-value less than 0.05. $p$-values for each gene and timepoint can be accessed via the GEO database (accession no. GSE119105, GSE119106, GSE119107)

greater abundance after $24 \mathrm{~h}$ of starvation of the $\Delta t h r A$ mutant (Fig. 3a). Furthermore, lysine, but not other pathway intermediates, was found to accumulate extracellularly (Fig. 3b), suggesting increased activity of a lysine exporter. Over a period of 4 days the extracellular concentration of lysine increased to 160 $\mu \mathrm{M}$ after which it stayed constant (Supplementary Fig. 2). Analysis of the metabolomic response of threonine auxotrophy alone $(M t b \Delta t h r B)$ showed a similar pattern of intracellular metabolite abundances (Fig. 4), but in addition, $M t b \Delta t h r B$ also accumulated homoserine (Supplementary Fig. 3), the adduct of the thrB encoded threonine kinase (Fig. 1a), which nicely confirms the metabolic block at ThrB. In line with this homoserine accumulation, we observed increased expression of $\operatorname{met} X$ (Fig. 2b). Lysine export was also observed in the $\Delta t h r B$ strain, albeit at a reduced rate compared to $\Delta t h r A$ (Supplementary Fig. 4), which fits with the fact that carbon rerouting in $\Delta t h r B$ occurs not only toward lysine but also homoserine. Homoserine was proposed to be toxic to $M$. tuberculosis ${ }^{20}$, and we have confirmed its toxicity for the MTB Complex strain M. bovis BCG (Supplementary Fig. 5). We observed an adverse role of homoserine but not threonine on growth of $M$. bovis BCG at concentrations above $50 \mu \mathrm{g} / \mathrm{ml}$ (Supplementary Fig. 5), which is in excellent agreement with the work published earlier ${ }^{20}$. These findings reveal accumulation of lysine, and homoserine in $\Delta t h r B$, that the cells attempt to manage by carbon-rerouting, lysine export and degradation. The lysine overload was particularly surprising given that most bacteria employ a lysine feedback loop that involves allosteric inhibition of aspartate kinase by lysine $e^{21-27}$.

Threonine is the main feedback regulator of $M t b$ 's aspartate pathway. Next, we wanted to understand at a regulatory level, how inhibition of ThrA or ThrB could lead to increased lysine production. Aspartate kinase (AK) encoded by ask (Rv3709c) catalyzes the first committed step in the aspartate pathway, converting aspartate to aspartyl phosphate (Fig. 1). In Escherichia coli, there are three $\mathrm{AK}$ isoforms that are separately sensitive to feedback regulation by threonine, lysine/leucine, or methionine, respectively $21,23,24$. The actinomycete Corynebacterium glutamicum regulates the aspartate pathway via a single $\mathrm{AK}$ that is feedback inhibited by lysine and threonine, and transcriptional repression of homoserine dehydrogenase $($ thr $A)$ by methionine $\mathrm{e}^{22,26}$. In M. tuberculosis, purified AK has been shown 
a

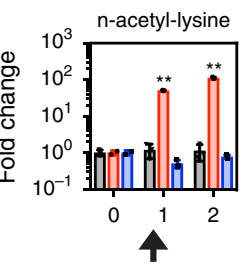

H37Rv
$\Delta t h r A$
$\Delta t h r A+$ L-threonine
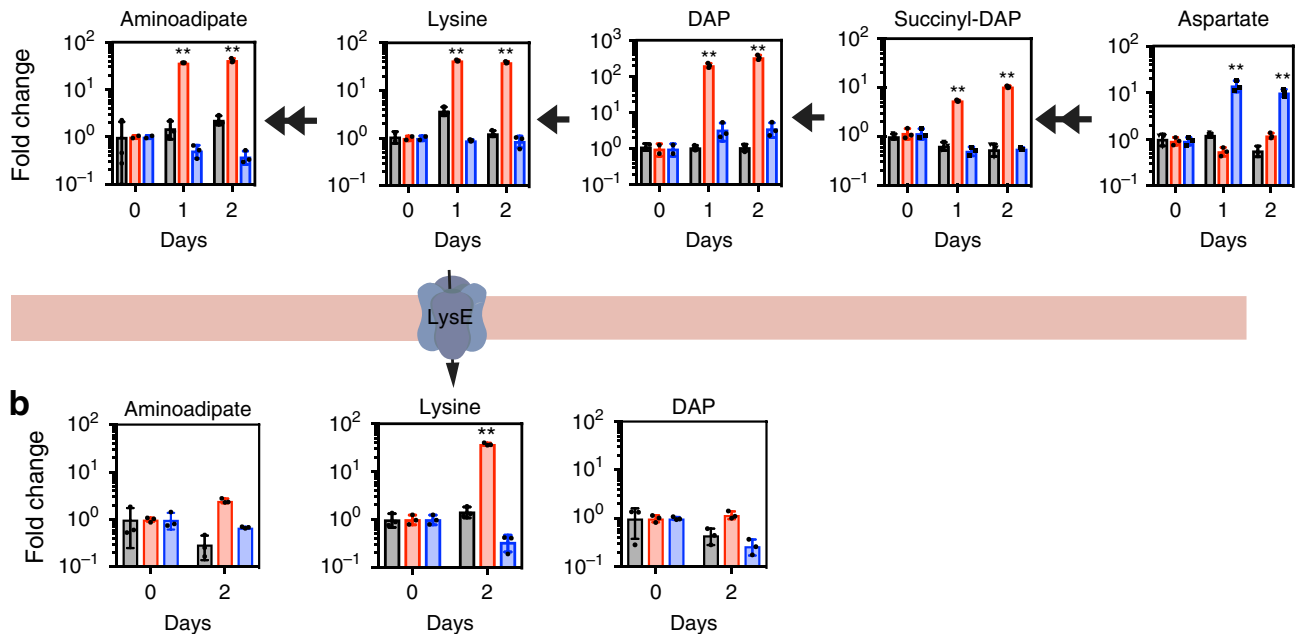

Fig. 3 Endo and exometabolome of Mtb $\Delta$ thrA. a Starvation of the homoserine auxotroph $M t b \Delta t h r A$ in unsupplemented medium (red bars) leads to strong (up to 100-fold) intracellular accumulation of lysine and DAP and its biosynthetic intermediates, as well as downstream metabolites aminoadipate, and nacetyl-lysine. Accumulation of the same intermediates is not observed in WT cells (Mtb H37Rv) under the same conditions (black bars). Addition of threonine to $M t b \Delta t h r A$ reverses accumulation of lysine and its intermediates (blue bars). Under the same conditions, aspartate but no other intermediate accumulates indicating that aspartate kinase is feedback inhibited by threonine. $\mathbf{b}$ Analysis of the exometabolome shows accumulation of lysine but no other intermediate in the supernatant of unsupplemented Mtb $\Delta$ thrA (red bars). Threonine addition stopped lysine export (blue bars). All values are the average of three biological replicates $(n=3) \pm$ s.d. (error bars depict standard deviation) and are representative of a minimum of two independent experiments. ${ }^{\star \star} p$-value $<0.01$ in Student's $t$-test. Bars are overlaid with dot plot of individual values. Source data are provided as a Source Data file

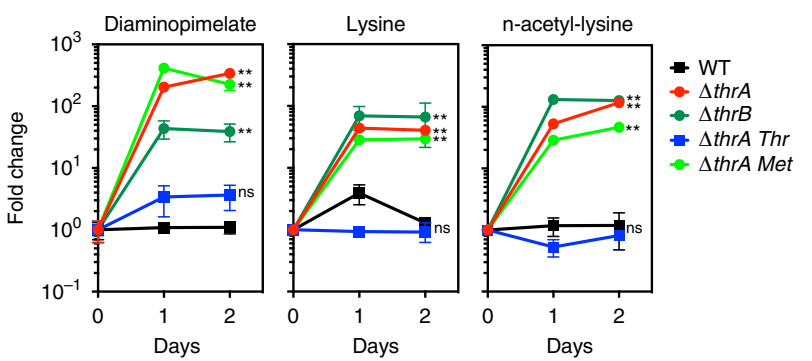

Fig. 4 Threonine but not methionine deficiency leads to lysine overload. To confirm that threonine deficiency alone is responsible for accumulation of lysine and intermediates, the endo-metabolome of the threonine auxotroph $M t b \Delta t h r B$ was determined and compared to Mtb $\Delta t h r A$. Accumulation of DAP, lysine and $n$-acetyl-lysine was detected in both strains. Only threonine but not methionine reversed lysine accumulation in Mtb $\Delta t h r A$. All values are the average of three biological replicates $(n=3) \pm$ s.d. (error bars depict standard deviation) and representative of two independent experiments. ${ }^{\star *} p$-value $<0.01$ in Student's $t$-test

to be sensitive to threonine but not lysine ${ }^{27}$, yet no other amino acids (e.g., methionine or homoserine) were tested nor was it investigated if these results translated to whole-cell physiology. Methionine and SAM biosynthesis in $M t b$ are likely regulated at the transcriptional level ${ }^{13,28}$. Consequently, addition of threonine should reverse lysine accumulation in $M t b \Delta t h r A$. Indeed, threonine was capable of decreasing intracellular lysine accumulation, causing a concomitant accumulation of aspartate
(Fig. 3a). Furthermore, addition of methionine as a control, caused no change in lysine pathway metabolites (Fig. 4), indicating that the loss of threonine is the major driver for overproduction of lysine. This argues that L-threonine inhibition of $\mathrm{AK}$ is likely a primary metabolic control mechanism of the aspartate pathway.

A lysine exporter responds to disruptions in lysine homeostasis. We next investigated the unexpected export of lysine as an alternative mechanism to cope with lysine overload in the cell. Our transcriptomic analysis of the $\Delta t h r A$ and $\Delta t h r B$ mutants showed a strong upregulation of a putative lysine exporter $R v 1986$ and arginine biosynthesis genes (Fig. 2a, b). Moreover, metabolomic data demonstrates that intracellular accumulation of Lys leads to export of both lysine and arginine (Arg) (Fig. 5a) but not other amino acids or metabolites (Supplementary Fig. 6). It has been shown in C. glutamicum that lysine accumulation is toxic, and that export of both Lys and Arg is carried out by the exporter LysE ${ }^{29,30}$. A bioinformatic analysis showed that $M t b$ encodes several proteins with homology to LysE of C. glutamicum, yet only Rv1986 is located adjacent to its canonical transcriptional regulator LysG (Rv1985c) (Fig. 5b) ${ }^{19}$. Hence, we hypothesized that Rv1986 serves the purpose of an overflow mechanism to prevent intracellular lysine overload. To test the role of LysE in amino acid export of $M t b$, we measured the ability of $M t b$ to export toxic amino acid analogs in the presence and absence of the LysE exporter. This is a commonly used strategy to characterize amino acid exporters in whole cells ${ }^{31}$. We deleted the genes lysE (Rv1986) and lys $(R v 1985 c)$ in $M t b$ H37Rv, resulting 

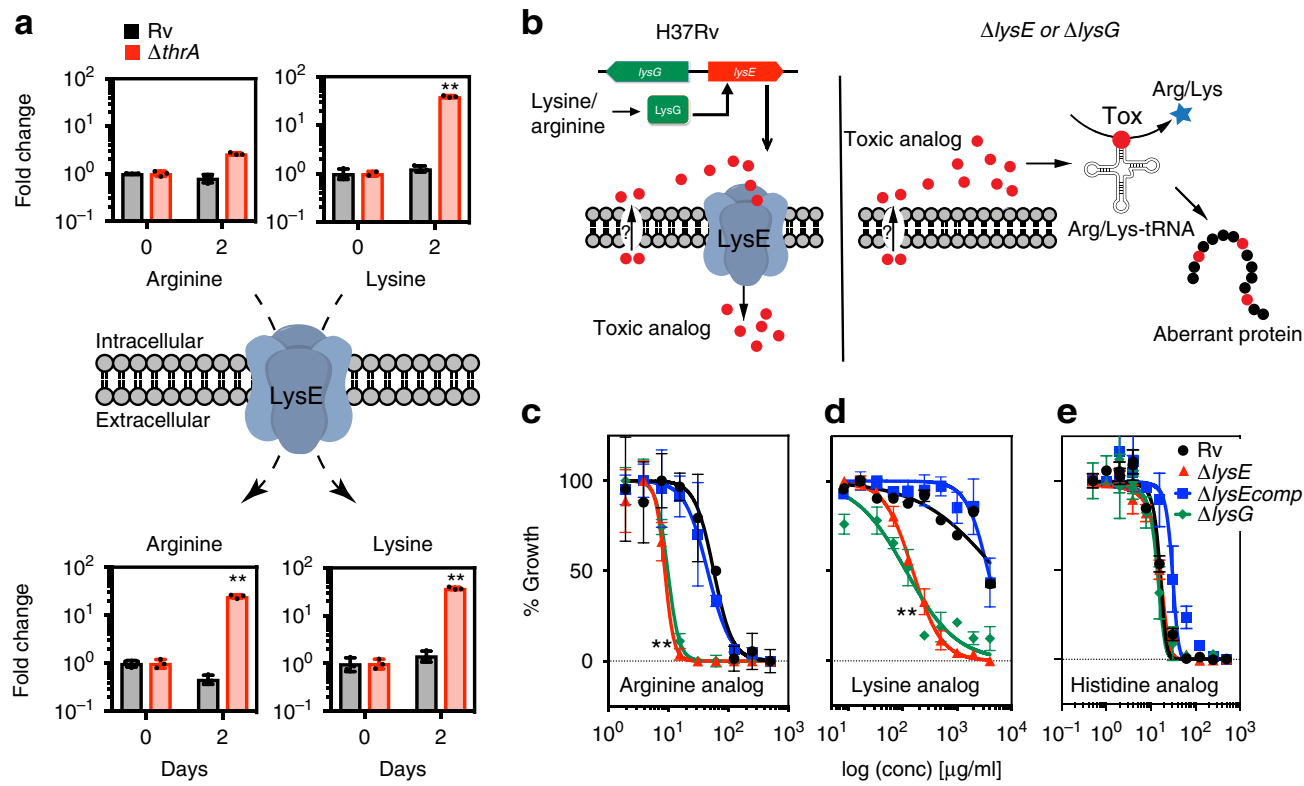

Fig. 5 Lysine and arginine are exported via the lysine permease LysE. a Accumulation of arginine and lysine in the supernatant of Mtb $\Delta$ thrA, indicated that Mtb harbors a lysine/arginine permease LysE. Rv1986 was identified bioinformatically as a LysE homolog and Rv1985c as its regulator LysG ${ }^{19}$. Both genes were individually deleted from Mtb H37Rv to yield strains $\Delta / y s E$ and $\Delta / y s G$ (Supplementary Table 1) and $\Delta / y s E$ was complemented with a plasmid containing lysE under its native promoter yielding strain $\Delta$ lysEcomp. b Illustration of how inactivation of LysE leads to hypersusceptibility to toxic analogs. c, d, e Exporter specificity for lysine and arginine was tested by MIC experiments on Mtb H37Rv (green), Mtb $\Delta$ lysE (blue), Mtb $\Delta$ lysEcomp (black) and Mtb $\Delta$ lys $G$ (red) using toxic analogs of $\mathbf{c}$ arginine (canavanine), $\mathbf{d}$ lysine (2,6-diaminohex-4-ynoic acid and) and $\mathbf{e}$ histidine (B-(2-Thiazolyl-DL-alanine). Mtb $\triangle l y s E$ and $\triangle$ lys $G$ are both hypersusceptible to toxic analogs of lysine and arginine but not histidine. All values are the average of three biological replicates $(n=3) \pm$ s.d. (error bars depict standard deviation) and are representative of a minimum of two independent experiments. ${ }^{\star \star} p$-value $<0.01$ in Student's $t$-test. Bars are overlaid with dot plot of individual values. Source data are provided as a Source Data file

in mutants $\Delta l y s E$ and $\Delta l y s G$. Using these strains, we determined the minimum inhibitory concentration (MIC) of toxic amino acid analogs of lysine (2,6-diaminohex-4-ynoic acid), arginine (canavanine) and histidine (ß-2-Thiazolyl-DL-alanine) (Fig. 5c, d, e). These compounds (Supplementary Fig. 1) have previously been shown to be toxic analogs in other bacteria and act as substrates for their cognate amino acid exporters ${ }^{32-34}$. Toxicity arises from their aberrant incorporation into proteins during translation, and deletion of their analogs' exporters should increase susceptibility (Fig. 5b). Indeed, we found that deletion of both $l y s E$ and $l y s G$ resulted in a greater than 10 -fold reduction in the MIC of canavanine and 2,6-diaminohex-4-ynoic acid (Fig. 5c, d), but there was no change in the MIC of the histidine analog control (Fig. 5e). The lysE phenotype was rescued when genetically complemented (Fig. 5c, d) or when the cognate amino acid (e.g., arginine) was added as a competitive substrate (Supplementary Fig. 7). The results demonstrate that LysE exports lysine and arginine as predicted by its homology and similarity (32\% identity determined by Basic Local Alignment Search Tool (BLAST)) to the C. glutamicum LysE permease and that LysG is the activator of LysE under the conditions tested (Fig. 5). We also found evidence that lysine disposal is relevant in $M t b$ during growth in standard growth medium under non-auxotrophic conditions. During the transition from exponential to stationary phase, lysine and aminoadipate are elevated in wild-type $M t b$ H37Rv (Supplementary Fig. 8) and the lysE deletion mutant shows a significant growth retardation under these conditions (Supplementary Fig. 9).

Taken together, these data indicate that $M t b$ utilizes lysine export and degradation pathways as a metabolic relief valve to prevent disruption of aspartate pathway homeostasis and maintain essential precursor production. Such a metabolic mechanism to maintain precursor availability for growth and survival is unprecedented in M. tuberculosis. It is tempting to speculate that prevention of lysine export, could synergize with inhibition of threonine production, and could be exploited to enhance bactericidal activity. To test this notion, we attempted to construct a double deletion strain $\Delta t h r A \Delta l y s E$. However, multiple attempts were unsuccessful pointing to a synthetic lethal interaction between these two pathways.

Aspartate pathway enzymes are required during chronic infections. Given the potent bactericidal phenotypes observed in several auxotrophs in vitro, we next evaluated the need for aspartate family amino acid biosynthesis during host infection. We first tested the ability of the threonine/methionine auxtotroph Mtb $\Delta$ thrA to establish an infection in immunocompetent $(\mathrm{C} 57 \mathrm{BL} / 6))$ and immunocompromised (SCID) mice via aerosol infection. Similar to $\Delta m e t X^{13}$, we found that $\Delta t h r A$ mutant was incapable of establishing an infection in either C57BL/6 (Supplementary Fig. 10a, b) or SCID mice (Supplementary Fig. 10c, d). To test whether threonine auxotrophy alone prevents the establishment of infection, we also tested $M t b \Delta t h r B$ in C57BL/6 and found this strain to be avirulent too (Supplementary Fig. 11). However, as $M t b$ can maintain a persistent infection it is also necessary to test target vulnerability during an established, chronic infection ${ }^{35}$. As specific target inhibitors are not yet available, a genetic approach was employed. We constructed conditional knockdowns (cKD) of ThrA ( $\Delta$ thrA-DUC) and MetX ( $\Delta$ metX-DUC), using the tetracycline-induced Dual-Control (DUC) system ${ }^{35}$. Both constructs are transcriptionally regulated by an anhydrotetracycline (atc) -inducible transcriptional repressor (T38), and are simultaneously repressed by atc-induced proteolytic degradation (DUC) ${ }^{36,37}$. The knockdown efficiency was validated in vitro by measuring the effect of increasing 
a

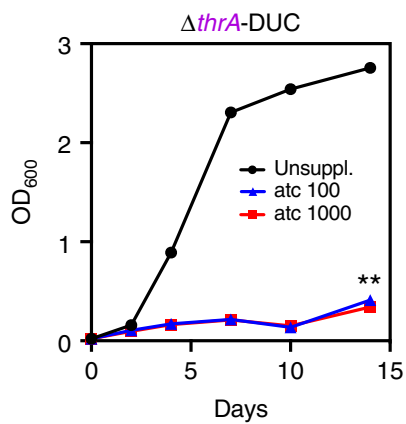

b

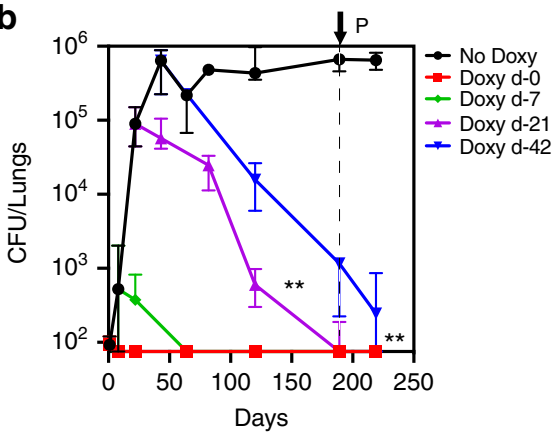

C

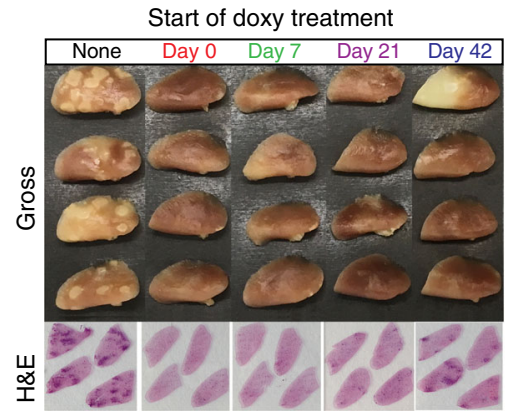

d

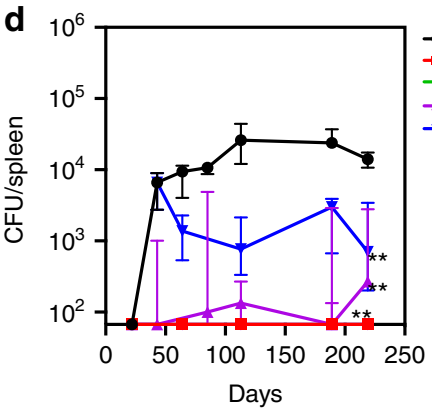

e

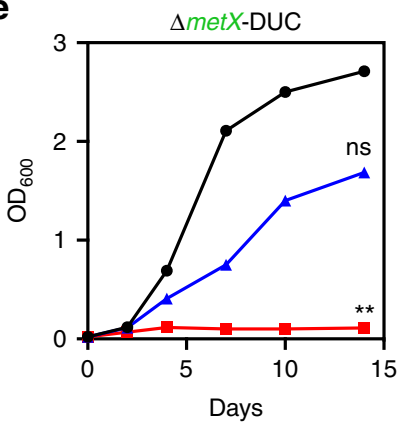

f

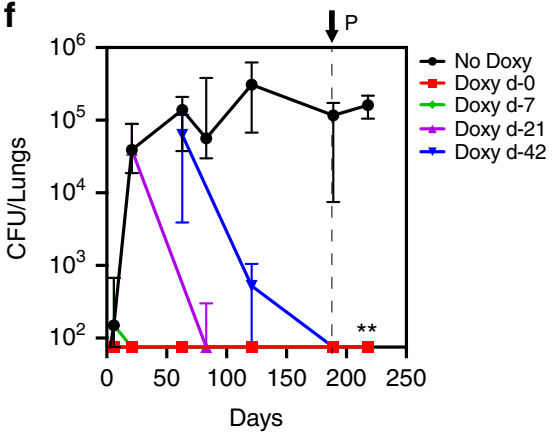

g

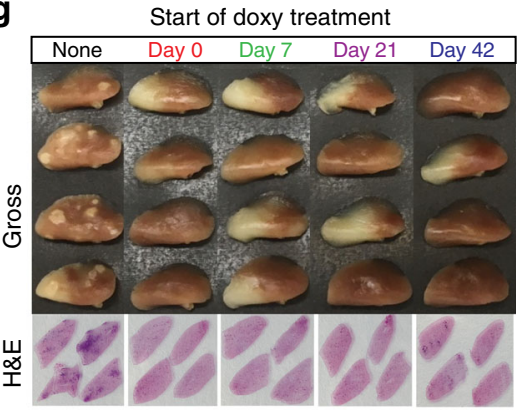

h

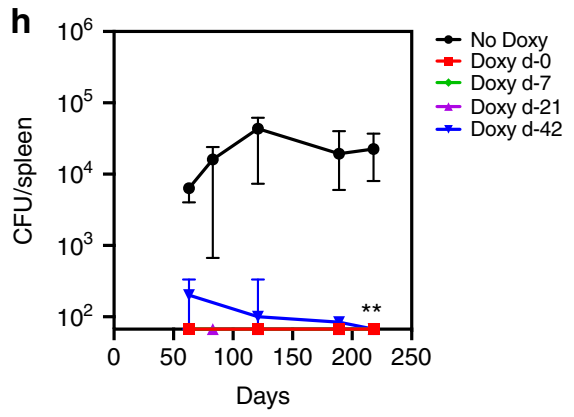

Fig. 6 Aspartate pathway enzymes are essential during acute and chronic mouse infections. Conditional knockdowns were constructed of two enzymes of the aspartate pathway: homoserine dehydrogenase ( $\Delta$ thrA-DUC) (a-d) and homoserine transacetylase ( $\Delta$ metX-DUC) (e-f). a, e Effect of gene knockdown/protein degradation on growth was tested in vitro in unsupplemented 7H9 OADC with different concentrations of anhydrotetracycline (atc). b, f Mice were aerosol infected with either $\triangle$ thrA-DUC3 or $\Delta$ met-DUC5 to deliver 100 CFU/lungs. Groups of mice were switched to doxycycline containing chow (2000 ppm) at day 0 (red square), day 7 (green diamond), day 21 (purple triangle) and day 42 (blue inverted triangle) to induce knockdown. One group (black) was left untreated throughout the whole experiment. At given time points, 4 mice per group were sacrificed and bacterial burden of lungs $(\mathbf{b}, \mathbf{f})$ and spleens ( $\mathbf{d}, \mathbf{h}$ ) determined by CFU counts of homogenized, serially diluted tissue. Gross lung pathology and Hematoxylin and Eosin (H\&E) staining of lungs at day 189 are shown under $(\mathbf{c}, \mathbf{g})$. $X$-axis crosses $Y$-axis at limit of detection. Error bars show standard deviations from $n=4$ mice. ${ }^{\star *} p$-value $<0.01,{ }^{*} p$-value $<0.05$ in Student's t-test. Source data are provided as a Source Data file

concentrations of atc on growth of the recombinant strains in culture broth media (Fig. 6a, e). Growth of the DUC strains in medium without atc was indistinguishable from the parental strain, demonstrating genetic complementation to WT levels (Supplementary Fig. 12a). While the parental H37Rv strain showed no sensitivity to atc (Supplementary Fig. 12b), growth was inhibited in both knockdown strains (Fig. 6a, b). Next we tested the vulnerability of $M t b$ to inhibition of these enzymes during different stages of infection in $\mathrm{C} 57 \mathrm{Bl} / 6$ mice. Cohorts of each infection group were started on doxycycline at $0,1,3$, or 6 weeks after infection, which allowed us to study the in vivo essentiality of these enzymes during both the acute and chronic 
a

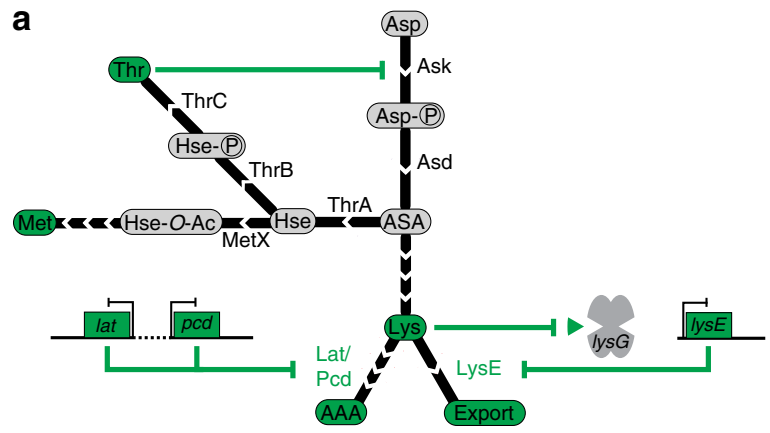

b

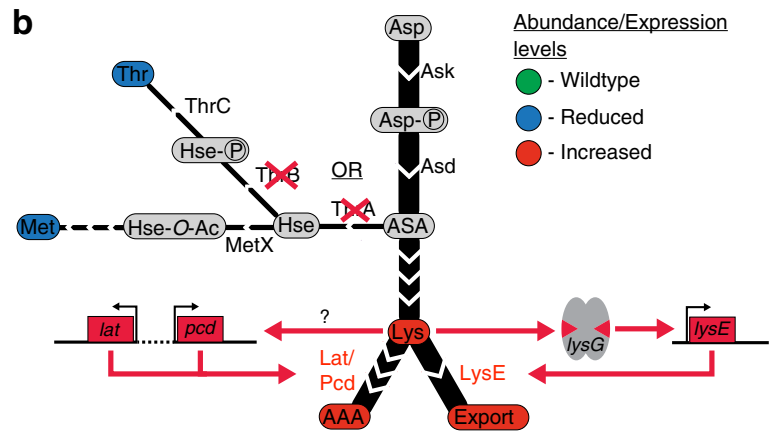

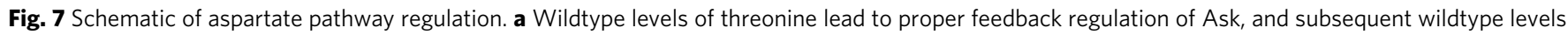
of lysine and its degradation (Lat/Pcd) and export (LysE) pathway enzymes and metabolites. b Disruption of threonine biosynthesis causes derepression of Ask leading to an accumulation of Lysine, which in turn activates the lysine degradation and export pathways. Nomenclature: Metabolites in rounded boxes: Asp: aspartate, Asp-P: aspartate-phosphate, ASA: aspartate-semialdehyde, Hse: Homoserine, Hse-P: homoserine-phosphate, Thr: Threonine, HsEO-Ac: O-acetyl-homoserine, Met: methionine, Lys: lysine, AAA: aminoadipate, Enzymes: Ask: aspartate kinase, Asd: aspartate semidaldehyde dehydrogenase, ThrA: homoserine dehydrogenase, ThrB: threonine kinase, ThrC: threonine synthase, MetX: homoserine O-acetyl-transferase, LysE: lysine exporter, Lat: lysine aminotransferase, Pcd: aminoadipate-semialdehyde dehydrogenase. Genes in square boxes. Color coding: green: wildtype levels, blue: reduces levels, red: increased levels

stages of infection (Fig. 6b, f). For both cKD strains, administration of doxycycline immediately after infection resulted in a total bacterial clearance (below detection limit) within one week after treatment initiation. Initiation of doxycycline treatment at the later time points resulted in clearance (below detection limit) of the infection from both lungs and spleens. By the end of the experiments, bacilli could no longer be cultured in the $\Delta m e t X$ DUC (Fig. 6f) infected mice, and only $\Delta$ thrA-DUC infected mice that began doxycycline treatment at week 6 still showed detectable levels of bacteria (Fig. 6b). The total bacterial burden had been reduced by 4 -logs in the lungs and $>1-\log$ in the spleens (Fig. 6d, h, respectively). Inspection of gross pathology and H\&E staining of lungs harvested 189 days after infection (indicated by arrow $\mathrm{P}$ and dotted line in Fig. 6b, f) reveal typical tuberculous lesions in untreated mice with high bacterial burden and absence of lesions and inflammatory foci in mice that were switched to doxy chow (Fig. 6c, g). These experiments demonstrate the essentiality of the aspartate pathway in both acute and chronic $M t b$ infections in animals and show that $M t b$ is unable to scavenge nutrients from the host to complement these auxotrophies in vivo.

\section{Discussion}

Significant efforts to identify new drug targets in $M t b$ have focused on the metabolism of essential nutrients. $M t b$ is an amino acid prototroph that has developed nominal machinery to obtain nutrients from the host, unlike many other intracellular parasites $^{12,38}$ and consequently, $M t b$ appears to be particularly susceptible to inhibition of amino acid biosynthesis ${ }^{12}$. Moreover, the biosynthetic enzymes for essential amino acids are largely absent in humans and mammals, making amino acid biosynthetic pathways in $M t b$ attractive drug targets ${ }^{12,39-42}$.

To date, in vitro transposon sequencing (Tn-seq) screens have been used to predict gene essentiality in $M t b^{43,44}$ and have guided target selection in drug discovery. The usefulness of Tn-seq screens for the TB research field is undisputed, yet their limitations for drug discovery need consideration. A recent study highlighted the impact of growth medium on inferred gene essentiality ${ }^{45}$ and illustrated how in vitro Tn-seq screens can be limited predictors of in vivo essentiality and cannot differentiate between lethality, bacteriostasis, slow growth phenotypes or conditional essentiality, all information that is crucial for assessing druggability. Since $M$. tuberculosis is an intracellular pathogen, essentiality and druggability of targets have to be tested during chronic infection in order to prevent false targets being taken forward for drug development.

We here focused on the aspartate pathway, because it produces multiple essential building blocks for vital cellular functions in $M t b$, such as cell wall biosynthesis, translation, and one-carbon metabolism. The rapid cell death phenotypes observed for auxotrophs of threonine (this study), homoserine (this study) and methionine ${ }^{13}$, points to a distinct susceptibility of $M t b$ to inhibition of this pathway. The mechanism of action for the lethality of threonine and homoserine auxotrophy appear to be multilayered. Cell death kinetics due to the loss of proteinogenic amino acids is likely accelerated by the accumulation of intermediates of lysine biosynthesis and homoserine (only in thrB mutant).

By studying the metabolic response to aspartate pathway inhibition, we discovered that $M t b$ employs a combination of feedback control, overflow metabolism and catabolic action to prevent flux imbalances and ensure balanced precursor production. We show here corroborating evidence that the committed step of the aspartate pathway in $M t b$, aspartate kinase, is controlled by allosteric feedback of threonine, but not by lysine or methionine. As $M t b$ lacks the ubiquitously used lysine-AK feedback loop, it must employ other regulatory mechanism to control production of proteinogenic lysine and the cell-wall building block diaminopimelate. By studying the adaptive metabolism of homoserine and threonine auxotrophic strains, we discovered two compensatory mechanisms that help $M t b$ to control the flux through this pathway branch: lysine degradation and export (Fig. 7b).

The lysine catabolic pathway catalyzed by Lat and Pcd is a unique feature of mycobacteria (Fig. 7). In a survey of 1362 bacteria, the aminoadipate branch was found to exist in only 36 microorganisms, predominately species of mycobacteria. This catabolic pathway seems to be a remnant of the cephamycin $\mathrm{C}$ biosynthetic pathway found in Streptomyces ${ }^{46}$, yet mycobacteria have only retained the first two reactions (mycobacteria do not produce cephamycin C). Another unique feature of this pathway in mycobacteria is the localization of lat in an operon with the alternative sigma factor $\operatorname{sigF}(\mathrm{Rv} 3286 \mathrm{c})$ and $\operatorname{lrp}(\mathrm{Rv} 3291 \mathrm{c})^{47}$ the putative transcriptional regulator of lat. SigF is important under various stress conditions, including osmotic stress, $\mathrm{pH}$, heatshock, oxidative stress ${ }^{48,49}$, and during infection ${ }^{50,51}$. Expression of $\operatorname{lrp}$ and lat genes is enriched in a persistence model ${ }^{17}$, as part of the stringent response ${ }^{16,18}$ and whenever $M t b$ transitions from replicating to non-replicating conditions (e.g., starvation or drug 
inhibition $)^{16,17}$. These observations fit well with the increased abundance of lysine and aminoadipate observed in stationary phase cells (Supplementary Fig. 8). It is tempting to speculate that $M t b$ has lost lysine feedback control at the AK level in order to allow increased lysine flux to act as a signal for cellular stress. In this model, elevated intracellular lysine levels would cause upregulation of the Lat degradation pathway, which generates aminoadipate and leads to the activation of the stringent response and persistence. Alternatively, lysine catabolism and its potential toxic intermediate(s) may serve as a means to control growth under conditions where replication could be lethal, similar to the role of Toxin-Antitoxin modules ${ }^{52}$ or methylglyoxal metabolism in persister cell formation ${ }^{53}$. Potential candidates for toxic intermediates are aminoadipate semialdehyde and piperideine-6carboxylate (Fig. 1a), as both have been shown to be cytotoxic ${ }^{54,55}$.

The second mechanism that mycobacteria employ to compensate for a missing lysine-AK feedback loop is catalyzed by a dedicated lysine/arginine permease LysE, encoded by Rv1986. We show that LysE in $M t b$ is used as a metabolic relief valve to clear excess cytoplasmic lysine, as a result of threonine auxotrophy. This exporter also has a role under non-auxotrophic conditions as evidenced by the retarded exit from lag phase and slow growth phenotype of the $M t b \Delta l y s E$ mutant. Current literature shows that Rv1986 is induced during infection of resting and activated macrophages $^{56}$, as well as in a lysosomal exposure model ${ }^{57}$ and it plays a role during latent $\mathrm{TB}$ infection in humans ${ }^{58}$. The exporter is a dominant target of IL-2 secreting memory T cells and is proposed to contribute to protective immunity in humans ${ }^{58}$. In fact, amino acids, specifically arginine, were shown to modulate human immunometabolism and T-cell responses ${ }^{59}$. Together, these data suggest that LysE-mediated export of arginine, and possibly lysine, might play a role in shaping $M t b$ 's niche during infection.

Our current knowledge of the nutritional composition of the $M t b$ microenvironment in the host is still incomplete. How that environment evolves over the course of an infection is even less understood $^{6,13,35}$. Auxotrophic bacterial strains are essential tools for identifying metabolic drug targets required during infection and probing the host environment for specific metabolites. However, because most $M t b$ auxotrophs fail to establish infection, their requirement for late-stage persistence cannot be investigated. To date, no data is available on the vulnerability of amino acid biosynthetic pathways during $M t b$ persistence in vivo. By utilizing conditional knockdowns of two branch-point enzymes ( $m e t X$ and $t h r A$ ), we uncovered the aspartate pathway as being broadly required for persistent infection in mice. These data also clearly demonstrate the inability of $M t b$ to scavenge sufficient methionine, homoserine or threonine from the host throughout the course of infection. The observations presented here lay an important foundation for future drug development. We propose that the numerous essential enzymes identified in this pathway and their absence in eukaryotic cells make it particularly amenable to drug discovery efforts.

\section{Methods}

Mycobacterial strain and growth conditions. All strains and plasmids used in this study are listed in Supplementary Tables 1 . Mycobacterial strains were grown in Middlebrook 7H9 medium (Difco) supplemented with $10 \%$ (vol/vol) OADC enrichment ( $0.5 \mathrm{~g}$ oleic acid, $50 \mathrm{~g}$ albumin, $20 \mathrm{~g}$ dextrose, $0.04 \mathrm{~g}$ catalase, $8.5 \mathrm{~g}$ sodium chloride in $1 \mathrm{~L}$ water), $0.2 \%$ ( vol/ $\mathrm{vol}$ ) glycerol, and $0.05 \%$ (vol/vol) tylaxopol (Sigma). Antibiotic selection media contained $75 \mu \mathrm{g} / \mathrm{mL}$ hygromycin B, $20 \mu \mathrm{g} /$ $\mathrm{mL}$ Kanamycin, and/or $25 \mu \mathrm{g} / \mathrm{mL}$ streptomycin, as required. Supplemented media for culturing auxotrophic mutants contained $50 \mu \mathrm{g} / \mathrm{mL}$ methionine, $500 \mu \mathrm{g} / \mathrm{mL}$ threonine, and/or $50 \mu \mathrm{g} / \mathrm{mL}$ homoserine. For time-course starvation and supplement growth curve experiments, strains were grown in the presence of the required supplements to an $\mathrm{OD}_{600}$ of 0.5 , washed three times with $\mathrm{PBS}+0.05 \%$ tylaxopol, and diluted into supplement free medium to an $\mathrm{OD}_{600}$ of 0.01 (growth/kill curves) or 0.2 (metabolomics and transcriptomics). Samples from three independent biological replicates were harvested on days $0,2,4$ and $7(\Delta t h r A)$ or days $0,1,2$ $(\Delta t h r B)$ for RNA extractions, and days 0,1 and 2 for metabolite extractions (see below). Chronologically we studied the $\Delta t h r A$ strain first and noticed that the main transcriptomic and metabolic changes are happening in the first 2 days. Hence, we focused on this timeframe for the rest of the study. Anhydrotetracycline (Acros) was dissolved in DMSO and used at the specified concentrations.

Gene Knockout and complementation. The genes thrA (Rv1294), thrB (Rv1296), lysE (Rv1986), and lysG (Rv1985c) were deleted in $M t b$ H37Rv by specialized transduction $^{60}$. The $M t b \mathrm{H} 37 \mathrm{Rv} \Delta m e t X$ strain (previously known as $\Delta m e t A$ ) was constructed previously ${ }^{13}$. Transductants were recovered on selective medium containing hygromycin $(75 \mu \mathrm{g} / \mathrm{mL})$ and appropriate amounts of supplements when needed: homoserine $(50 \mu \mathrm{g} / \mathrm{mL})$ for $M t b \Delta t h r A$ and threonine $(500 \mu \mathrm{g} / \mathrm{mL})$ for $M t b$ $\Delta t h r B$. Mutations were confirmed by three-primer PCR using respective $\mathrm{L}, \mathrm{R}$, and Universal_uptag primers, listed in Supplementary Table 2. The H37Rv $\Delta m e t X$ and $\Delta t h r A$ strains were complemented using the tetracycline-inducible dual-control (DUC) knockdown plasmids ${ }^{61}$. Briefly, each strain was complemented with pGMCK-q19-T38S38-P750-yfg-DAS+4 (pGMCK- $y f g$ ). The gene sequences were PCR-amplified using the respective $\mathrm{P} 1$ and $\mathrm{P} 2$ primers (Supplementary Table 2) and were recombined into pDO12A using BP clonase (Invitrogen), to generate the entry vectors pEN12A- $y f g$-DAS +4 . The final construct was generated by 3-way gateway cloning with pEN23A- $y f g$-DAS +4 , pEN41A-T38S38, pEN12A-P750, and pDE43-MCK using LR clonase II (Invitrogen), to generate the final construct. This was transformed into the respective mutant strain to generate DAS complements whose expression of the complemented genes was under a TetOFF promoter. The H37Rv metX-DAS and thrA-DAS strains were further transformed with pGMCtKq28-TSC10M1-sspB to introduce Tet-inducible degradation of DAS +4 tagged proteins. Shine-delgarno sequences were mutated according to Supplementary Table 3, using Phusion Site-directed Mutagenesis kits (NEB). The H37Rv $\Delta l y s E$ strain was complemented by transformation with pMV361 harboring a copy of Rv1986 (Supplementary Table 2). This DNA fragment was PCR amplified using primers Rv1986_fw_EcoR1 and Rv1986_Re_HindIII (Supplementary Table 2) and was cloned into pMV361 using EcoRI and HindIII restriction sites, resulting in plasmid pMV361-LysE

Metabolite extractions. Samples of bacterial cultures in biological triplicates were harvested at given time points ${ }^{13,62}$. For intracellular metabolites, an equivalent of $10 \mathrm{~mL}$ culture at an $\mathrm{OD}_{600}$ of 0.2 was rapidly filtered on a $0.22 \mu \mathrm{m}$ nitrocellulose filter (Millipore). The filter papers were immediately placed in 2-mL screw cap tubes of $1 \mathrm{~mL}$ extraction solvent containing 20\%/40\%/40\% (vol/vol) water/acetonitrile/methanol (Fisher) with approximately $500 \mu \mathrm{L}$ of silica beads at $-20{ }^{\circ} \mathrm{C}$. The samples were homogenized twice in a Precellys Evolution (Bertin) at 4,500 rpm for 45 seconds, cooled to $5^{\circ} \mathrm{C}$ using a Cryolys (Bertin), and with 5 min rest between cycles. Samples were centrifuged, and $750 \mu \mathrm{L}$ of the extract was filtered through $0.22-\mu \mathrm{m}$ Spin-X centrifuge filters (Corning, Life Technologies) at 10,621 g for 3 min, and stored at $-80^{\circ} \mathrm{C}$. For extracellular metabolites, $1 \mathrm{~mL}$ of culture was collected and centrifuged, and $750 \mu \mathrm{L}$ of supernatant was filtered twice through $0.22-\mu \mathrm{m}$ Spin-X columns. Detergent in the samples were removed using Pierce detergent removal spin columns (Thermo), according to manufacturer's instructions. Metabolites were extracted by adding $200 \mu \mathrm{L}$ sample to $800 \mu \mathrm{L}$ of $50 \% / 50 \%$ ( $\mathrm{vol} / \mathrm{vol}$ ) acetonitrile/methanol (Fisher), the samples were spun down, as before, and the supernatant collected and stored at $-80^{\circ} \mathrm{C}$ until analysis.

Metabolomics. Metabolomics analysis was performed using an Acquity UPLC system coupled with a Synapt G2 quadrupole-time of flight hybrid mass spectrometer (Waters, Massachusetts, USA) ${ }^{13}$. Column eluents were delivered via electrospray ionization. UPLC was performed in a hydrophilic interaction liquid chromatography-mode (HILIC) gradient elution using an Acquity $1.7-\mu \mathrm{m}$ amide column $(2.1 \times 100 \mathrm{~mm})^{63}$. The flow rate is $0.5 \mathrm{~mL} / \mathrm{min}$ with mobile phase A $(100 \%$ acetonitrile) and mobile phase B (100\% water), both containing $0.1 \%$ formic acid In both positive and negative mode, the gradient began with $1 \% \mathrm{~B}$ until $1 \mathrm{~min}$, ramped to $35 \% \mathrm{~B}$ by $14 \mathrm{~min}$, then $60 \% \mathrm{~B}$ by $17 \mathrm{~min}$, held at $60 \% \mathrm{~B}$ for $1 \mathrm{~min}$, then ramped to $1 \% \mathrm{~B}$ by $19 \mathrm{~min}$ and held at $1 \% \mathrm{~B}$ to the end of run at $20 \mathrm{~min}$. The mass spectrometer was operated in $\mathrm{V}$ mode for high sensitivity using a capillary voltage of $2 \mathrm{kV}$ and a cone voltage of $17 \mathrm{~V}$. The desolvation gas flow rate was $500 \mathrm{~L} / \mathrm{h}$, and the source and desolvation gas temperature were $120^{\circ} \mathrm{C}$ and $325^{\circ} \mathrm{C}$, respectively. MS spectra were acquired in centroid mode from $\mathrm{m} / \mathrm{z} 50-1,200$ with a scan time of $0.5 \mathrm{~s}$. Leucine enkephalin $(2 \mathrm{ng} / \mu \mathrm{L})$ was used as lock mass $(\mathrm{m} / z 556.2771$ and 554.2615 in positive and negative experiments, respectively). Analysis was performed using MarkerLynx (Waters) with extended statistic function to identify statistically significant differences of metabolite abundances. A TargetLynx (Waters) database was compiled by running standards of metabolites of interest to determine retention times and $\mathrm{m} / \mathrm{z}$ ratios. Calibration curves of compound mixes are run routinely to determine the linear range of metabolite detection. All samples were analyzed using TargetLynx with manual curation of peak areas where necessary. With abundances we refer to are raw intensity values for the area under the curve for each metabolite peak. Peak areas were used to calculate fold changes of metabolites relative to day 0 . Metabolite abundances, retention times and $\mathrm{m} / \mathrm{z}$ values can be found in Supplementary Data 1. Threonine and homoserine have the 
same molecular mass. The retention time of homoserine is $0.1-0.2 \mathrm{~min}$ slower than threonine, however differentiation of the two peaks is difficult. Fragmentation pattern of the two compounds in positive mode is nearly identical while in negative mode there is one distinction $(\mathrm{m} / \mathrm{z} 72.04)$. We have detected high amounts of $\mathrm{m} / \mathrm{z}$ $=120.0655$ in the ThrB strain but not in Rv or ThrA. Since ThrB is downstream of homoserine, we hypothesize that this mass must represent homoserine, because the bacterium does not grow without the addition of high concentration threonine. In the ThrA strain, as expected from a strain that cannot produce homoserine nor threonine, the $\mathrm{m} / \mathrm{z} 120.0655$ was undetectable.

RNA extraction. Samples of bacterial cultures were harvested in triplicate at appropriate times. $10 \mathrm{~mL}$ of culture was pelleted and the supernatant decanted. The cell pellet was resuspended in $1 \mathrm{~mL}$ of TRIzol (Invitrogen) and incubated overnight at $4{ }^{\circ} \mathrm{C}$, before storage at $-80^{\circ} \mathrm{C}$. The suspension was transferred to Fast-Prep Blue Cap tubes and processed twice for $45 \mathrm{~s}$ at speed 6 in a Fast-Prep apparatus (MP Biomedicals). After a brief incubation on ice, the debris was spun down, and the supernatant $(750 \mu \mathrm{L})$ was processed for purification (described below).

Microarray (Mtb $\Delta$ thrA). Total RNA was purified from the cell lysate using Direct-zol RNA MiniPrep (Zymo Research) with on-column DNase I according to the manufacturer's protocol. Total RNA $(2 \mu \mathrm{g})$ was used to create cDNA labeled with aminoallyl dUTP (Sigma). Fluorescent Cy3 and Cy5 dyes (GE Healthcare) then were covalently attached to the aminoallyl tags. Each pair of differentially labeled probes was resuspended in $60 \mu \mathrm{L}$ of hybridization buffer $(500 \mu \mathrm{L}$ formamide, $250 \mu \mathrm{L} 20 \times \mathrm{SSC}, 5 \mu \mathrm{L} 10 \%$ (wt/vol) SDS, $245 \mu \mathrm{L}$ ultrapure water) and hybridized to the microarray slide overnight in a $42{ }^{\circ} \mathrm{C}$ water bath. Slides then were washed in increasingly stringent wash conditions (three times for $5 \mathrm{~min}$ each washing in $1 \times$ SSC $0.1 \%$ SDS; three times $5 \mathrm{~min}$ each washing in $0.1 \times$ SSC $0.1 \%$ SDS; three times for 5 min each washing in $0.1 \times$ SSC, and a final dip in 0.22 $\mu \mathrm{m}$-filtered Milli-Q water [Millipore]). Arrays were scanned in a Genepix 4000A scanner, and spots were quantified with TIGR Spotfinder. The data then were processed in TIGR MIDAS. Two-color tiff images of the microarrays were inspected visually for defects and obvious spatial biases. Before normalization, pintip (block) intensity box plots were used to detect slides exhibiting spatial and/or pin biases; after normalization, intensity box plots and MA plots were used to assess the effectiveness of normalization in correcting these biases. Radio-intensity plots were used to identify outlier slides and to assess the effectiveness of the normalization process, and Z-score histograms were used to look for slides with abnormal intensity distributions. Slides that did not pass the quality-control process were rejected, and repeat hybridizations were performed. After normalization (total intensity and LOWESS) the in-slide replicate spots were averaged before expression ratios were calculated. The results from four independent biological replicates including two dye swaps then were subjected to a t-test without false discovery correction in TIGR MeV software. The analysis was used as a ranking method. For a general overview, genes with expression ratios $>2$ and $<0.5$ and a Pvalue $<0.05$ were used for data interpretation.

RNA-seq (Mtb $\Delta$ thrB). RNA from the clarified cell lysate samples were purified by Trizol $^{\circledast}$-chlorofom precipitation according to the manufacturer instructions (Invitrogen). After purification remaining DNA was removed from the samples using the DNA-Free Turbo DNase Kit (Ambion) and RNA integrity was checked on a Bioanalyzer RNA 6000 Pico chip (Agilent). The ribosomal RNA (rRNA) was removed using the Bacterial RiboZero kit (Illumina), and removal was confirmed by Bioanaylzer as above. RNA-seq library preparation was conducted with the TrueSeq Stranded mRNA Library Prep (Illumina) according to manufacturer's instructions and the library was sequenced on a NextSeq 500 (Illumina) using the NextSeq 500/550 HO V2 (75 cycles) (Illumina). Illumina reads were mapped to the M. tuberculosis reference genome from NCBI (NC_000962.3) in the form of a SAM file using Bowtie2 (http://bowtie-bio.sourceforge.net/index.shtml), the SAM files were converted to coordinate-sorted BAM files using samtools, and reads over genes were counted using featureCounts ${ }^{64}$. Differential expression analysis was then conducted using $R$ and the DESeq2 package.

Mouse experiments. Mouse studies were performed in accordance with National Institutes of Health guidelines following the recommendations in the Guide for the Care and Use of Laboratory Animals ${ }^{65}$. The protocols used in this study were approved by the Institutional Animal Care and Use Committee of Albert Einstein College of Medicine (Protocols \#20120114 and \#20180612).

Female SCID mice and female C57BL/6 mice (Jackson Laboratories) were infected via the aerosol route using a $1 \times 10^{7} \mathrm{cfu} / \mathrm{mL}$ mycobacterial suspension in PBS containing $0.05 \%$ tylaxopol and $0.004 \%$ antifoam. Infection yielded approximately 100 bacilli per lung as determined by quantification of lung bacterial loads at $24 \mathrm{~h}$ post-infection (four mice per group). Subsequently, four mice from each group were killed at days 1, 7, 21, 63, 84, 98 (C57BL/6) or 1, 14, 22, and 42 (SCID) to determine the bacterial burden in the lung and spleen. Six SCID mice per group were kept for survival experiments. For conditional knockdown experiments, groups of mice infected with each strain were started on $2000 \mathrm{ppm}$ doxycyclinetreated chow (TestDiet, Missouri, USA) at 0, 1, 3, and 6 weeks. Four mice from each group were sacrificed at given times, the lungs and spleens were harvested, homogenized and plated on 7H10 media containing the appropriate supplement to determine the bacterial burden. All mice infected with $M t b$ were maintained under appropriate conditions in an animal biosafety level 3 laboratory.

Pathology. Lung samples were fixed in 10\% neutral buffered formalin for $48-72 \mathrm{~h}$ and the subjected to paraffin embedment. Tissues were sectioned at $5 \mu \mathrm{m}$ and stained with Hematoxylin and Eosin (H\&E).

MIC determination of toxic metabolite analogs. Cultures of $M t b \mathrm{H} 37 \mathrm{Rv}, \Delta l y s E$, $\Delta l y s E c o m p$, and $\Delta l y s G$ were grown to log-phase in unsupplemented media. Each toxic analog (Supplementary Fig. 1) was two-fold serially diluted in a sterile 96 well plate. In triplicate, each strain was diluted to an $\mathrm{OD}_{600}$ of 0.001 in wells containing each of the drugs to a final volume of $0.2 \mathrm{~mL}$. The plates were incubated at $37^{\circ} \mathrm{C}$ for 7 days and the growth was measured by optical density in an Epoch plate reader (Biotek, Vermont, USA). Percent growth was calculated based on the density in the untreated wells. For arginine rescue experiments, arginine was added to each well at a concentration of $50 \mu \mathrm{g} / \mathrm{ml}$.

Reporting summary. Further information on research design is available in the Nature Research Reporting Summary linked to this article.

\section{Data availability}

All microarray and RNAseq raw data were deposited in Gene Expression Omnibus (GEO) (GSE119105 ( $\Delta t h r A)$, GSE119106 ( $\Delta t h r B)$, GSE119107 (SuperSeries)). Data underlying Figs. 1-6 are provided as Source Data files. All other data are available from the corresponding author upon reasonable requests.

Received: 14 September 2018 Accepted: 28 August 2019

Published online: 16 September 2019

\section{References}

1. WHO. Global Tuberculosis Report 2017. (Geneva, Switzerland, 2017).

2. Dartois, V. The path of anti-tuberculosis drugs: from blood to lesions to mycobacterial cells. Nat. Rev. Microbiol 12, 159-167 (2014).

3. Connolly, L. E., Edelstein, P. H. \& Ramakrishnan, L. Why is long-term therapy required to cure tuberculosis? PLoS Med. 4, e120 (2007).

4. Rhee, K. Y. et al. Central carbon metabolism in Mycobacterium tuberculosis: an unexpected frontier. Trends Microbiol 19, 307-314 (2011).

5. Ehrt, S., Schnappinger, D. \& Rhee, K. Y. Metabolic principles of persistence and pathogenicity in Mycobacterium tuberculosis. Nat. Rev. Microbiol 16, 496-507 (2018)

6. Warner, D. F. Mycobacterium tuberculosis metabolism. Cold Spring Harb. Perspect. Med. 5, pii: a021121 (2014).

7. Ruecker, N. et al. Fumarase deficiency causes protein and metabolite succination and intoxicates Mycobacterium tuberculosis. Cell Chem. Biol. 24, 306-315 (2017).

8. Trujillo, C. et al. Triosephosphate isomerase is dispensable in vitro yet essentia for Mycobacterium tuberculosis to establish infection. MBio 5, e00085 (2014).

9. Puckett, S. et al. Inactivation of fructose-1,6-bisphosphate aldolase prevents optimal co-catabolism of glycolytic and gluconeogenic carbon substrates in Mycobacterium tuberculosis. PLoS Pathog. 10, e1004144 (2014).

10. Puckett, S. et al. Glyoxylate detoxification is an essential function of malate synthase required for carbon assimilation in Mycobacterium tuberculosis. Proc. Natl. Acad. Sci. USA 114, E2225-E2232 (2017).

11. Eoh, H. et al. Metabolic anticipation in Mycobacterium tuberculosis. Nat. Microbiol 2, 17084 (2017)

12. Berney, M. \& Berney-Meyer, L. Mycobacterium tuberculosis in the Face of Host-Imposed Nutrient Limitation. Microbiol Spectr. 5, TBTB2-0030-2016 (2017).

13. Berney, M. et al. Essential roles of methionine and S-adenosylmethionine in the autarkic lifestyle of Mycobacterium tuberculosis. Proc. Natl Acad. Sci. USA 112, 10008-10013 (2015)

14. Pavelka, M. S. Jr. \& Jacobs, W. R. Jr. Biosynthesis of diaminopimelate, the precursor of lysine and a component of peptidoglycan, is an essential function of Mycobacterium smegmatis. J. Bacteriol. 178, 6496-6507 (1996).

15. Viola, R. E. The central enzymes of the aspartate family of amino acid biosynthesis. Acc. Chem. Res. 34, 339-349 (2001).

16. Betts, J. C., Lukey, P. T., Robb, L. C., McAdam, R. A. \& Duncan, K. Evaluation of a nutrient starvation model of Mycobacterium tuberculosis persistence by gene and protein expression profiling. Mol. Microbiol 43, 717-731 (2002).

17. Keren, I., Minami, S., Rubin, E. \& Lewis, K. Characterization and transcriptome analysis of Mycobacterium tuberculosis persisters. MBio 2, e00100-e00111 (2011) 
18. Duan, X. et al. Mycobacterium Lysine epsilon-aminotransferase is a novel alarmone metabolism related persister gene via dysregulating the intracellular amino acid level. Sci. Rep. 6, 19695 (2016).

19. Schneefeld, M., Busche, T., Geffers, R., Kalinowski, J. \& Bange, F. C. The transcriptional regulator LysG (Rv1985c) of Mycobacterium tuberculosis activates lysE (Rv1986) in a lysine-dependent manner. PLoS ONE 12, e0186505 (2017).

20. O'Barr, T. P. \& Everett, K. A. Effect of 1-homoserine on the growth of Mycobacterium tuberculosis. Infect. Immun. 3, 328-332 (1971).

21. Stadtman, E. R. Symptosium on multiple forms of enzymes and control mechanisms. II. Enzyme multiplicity and function in the regulation of divergent metabolic pathways. Bacteriol. Rev. 27, 170-181 (1963).

22. Cremer, J., Treptow, C., Eggeling, L. \& Sahm, H. Regulation of enzymes of lysine biosynthesis in Corynebacterium glutamicum. J. Gen. Microbiol 134, 3221-3229 (1988).

23. Atkinson, D. E. Regulation of enzyme function. Annu Rev. Microbiol 23, 47-68 (1969).

24. Patte, J. C., Le Bras, G. \& Cohen, G. N. Regulation by methionine of the synthesis of a third aspartokinase and of a second homoserine dehydrogenase in Escherichia coli K 12. Biochim Biophys. Acta 136, 245-247 (1967).

25. Nakayama, K., Kitada, S. \& Kinoshita, S. Studies on lysine fermentation I. The control mechanism on lysine accumulation by homoserine and threonine. J. Gen. Appl. Microbiol. 7, 145-154 (1961).

26. Nakayama, K., Tanaka, H., Hagino, H. \& Kinoshita, S. Studies on lysine fermentation Part V. Concerted feedback inhibition of aspartokinase and the absence of lysine inhibition on aspartic semialdehyde-pyruvate condensation in micrococcus glutamicus. Agric. Biol. Chem. 30, 611-616 (1966).

27. Yang, Q. et al. Structural view of the regulatory subunit of aspartate kinase from Mycobacterium tuberculosis. Protein Cell 2, 745-754 (2011).

28. Weinberg, Z. et al. The aptamer core of SAM-IV riboswitches mimics the ligand-binding site of SAM-I riboswitches. RNA 14, 822-828 (2008).

29. Bellmann, A. et al. Expression control and specificity of the basic amino acid exporter LysE of Corynebacterium glutamicum. Microbiology 147, 1765-1774 (2001).

30. Erdmann, A., Weil, B. \& Kramer, R. Lysine secretion by wild-type Corynebacterium glutamicum triggered by dipeptide uptake. J. Gen. Microbiol 139, 3115-3122 (1993).

31. Nandineni, M. R. \& Gowrishankar, J. Evidence for an arginine exporter encoded by yggA ( $\operatorname{argO})$ that is regulated by the LysR-type transcriptional regulator ArgP in Escherichia coli. J. Bacteriol. 186, 3539-3546 (2004).

32. Cai, T. et al. Host legume-exuded antimetabolites optimize the symbiotic rhizosphere. Mol. Microbiol 73, 507-517 (2009).

33. Davis, A. L., Lloyd, R., Maul, S., Cook, D. E. \& McCord, T. J. 2,6-Diamino-4hexynoic acid, a lysine analog. Arch. Biochem Biophys. 104, 238-240 (1964).

34. Kulis-Horn, R. K., Persicke, M. \& Kalinowski, J. Corynebacterium glutamicum ATP-phosphoribosyl transferases suitable for L-histidine productionstrategies for the elimination of feedback inhibition. J. Biotechnol. 206, 26-37 (2015).

35. Kim, J. H. et al. A genetic strategy to identify targets for the development of drugs that prevent bacterial persistence. Proc. Natl Acad. Sci. USA 110, 19095-19100 (2013).

36. Woong Park, S. et al. Evaluating the sensitivity of Mycobacterium tuberculosis to biotin deprivation using regulated gene expression. PLoS Pathog. 7, e1002264 (2011).

37. Wei, J. R. et al. Depletion of antibiotic targets has widely varying effects on growth. Proc. Natl Acad. Sci. USA 108, 4176-4181 (2011).

38. Zhang, Y. J. \& Rubin, E. J. Feast or famine: the host-pathogen battle over amino acids. Cell. Microbiol. 15, 1079-1087 (2013).

39. Evans, J. C. \& Mizrahi, V. The application of tetracyclineregulated gene expression systems in the validation of novel drug targets in Mycobacterium tuberculosis. Front Microbiol 6, 812 (2015).

40. Wellington, S. et al. A small-molecule allosteric inhibitor of Mycobacterium tuberculosis tryptophan synthase. Nat. Chem. Biol. 13, 943-950 (2017).

41. Zhang, Y. J. et al. Tryptophan biosynthesis protects mycobacteria from CD4 T-cell-mediated killing. Cell 155, 1296-1308 (2013).

42. Wellington, S. \& Hung, D. T. The Expanding Diversity of Mycobacterium tuberculosis Drug Targets. ACS Infect. Dis. 4, 696-714 (2018).

43. DeJesus, M. A., et al. Comprehensive essentiality analysis of the Mycobacterium tuberculosis Genome via saturating transposon mutagenesis. MBio 8, pii: e02133-16 (2017).

44. Sassetti, C. M., Boyd, D. H. \& Rubin, E. J. Genes required for mycobacterial growth defined by high density mutagenesis. Mol. Microbiol 48, 77-84 (2003).

45. Minato, Y., et al. Genomewide assessment of Mycobacterium tuberculosis conditionally essential metabolic pathways. mSystems 4, pii: e00070-19 (2019).

46. Liras, P. Biosynthesis and molecular genetics of cephamycins. Cephamycins produced by actinomycetes. Antonie Van. Leeuwenhoek 75, 109-124 (1999).
47. Neshich, I. A., Kiyota, E. \& Arruda, P. Genome-wide analysis of lysine catabolism in bacteria reveals new connections with osmotic stress resistance. ISME J. 7, 2400-2410 (2013).

48. Gebhard, S., Humpel, A., McLellan, A. D. \& Cook, G. M. The alternative sigma factor SigF of Mycobacterium smegmatis is required for survival of heat shock, acidic $\mathrm{pH}$ and oxidative stress. Microbiology 154, 2786-2795 (2008).

49. Humpel, A., Gebhard, S., Cook, G. M. \& Berney, M. The SigF regulon in Mycobacterium smegmatis reveals roles in adaptation to stationary phase, heat and oxidative stress. J. Bacteriol. 192, 2491-2502 (2010).

50. Chen, P., Ruiz, R. E., Li, Q., Silver, R. F. \& Bishai, W. R. Construction and characterization of a Mycobacterium tuberculosis mutant lacking the alternate sigma factor gene, sigF. Infect. Immun. 68, 5575-5580 (2000).

51. Karls, R. K., Guarner, J., McMurray, D. N., Birkness, K. A. \& Quinn, F. D. Examination of Mycobacterium tuberculosis sigma factor mutants using lowdose aerosol infection of guinea pigs suggests a role for SigC in pathogenesis. Microbiology 152, 1591-1600 (2006).

52. Sala, A, Bordes, P. \& Genevaux, P. Multiple toxin-antitoxin systems in Mycobacterium tuberculosis. Toxins (Basel). 6, 1002-1020 (2014).

53. Girgis, H. S., Harris, K. \& Tavazoie, S. Large mutational target size for rapid emergence of bacterial persistence. Proc. Natl Acad. Sci. USA 109 12740-12745 (2012).

54. Zaret, K. S. \& Sherman, F. alpha-Aminoadipate as a primary nitrogen source for Saccharomyces cerevisiae mutants. J. Bacteriol. 162, 579-583 (1985).

55. Mills, P. B. et al. Mutations in antiquitin in individuals with pyridoxinedependent seizures. Nat. Med. 12, 307-309 (2006).

56. Schnappinger, D. et al. Transcriptional adaptation of Mycobacterium tuberculosis within macrophages: insights into the phagosomal environment. J. Exp. Med. 198, 693-704 (2003).

57. Lin, W. et al. Transcriptional profiling of Mycobacterium tuberculosis exposed to in vitro lysosomal stress. Infect. Immun. 84, 2505-2523 (2016).

58. Gideon, H. P. et al. Hypoxia induces an immunodominant target of tuberculosis specific T cells absent from common BCG vaccines. PLoS Pathog. 6, e1001237 (2010).

59. Geiger, R. et al. L-Arginine modulates $\mathrm{T}$ cell metabolism and enhances survival and anti-tumor activity. Cell 167, 829-842 e813 (2016).

60. Jain, P. et al. Specialized transduction designed for precise high-throughput unmarked deletions in Mycobacterium tuberculosis. MBio 5, e01245-01214 (2014).

61. Schnappinger, D., O'Brien, K. M. \& Ehrt, S. Construction of conditional knockdown mutants in mycobacteria. Methods Mol. Biol. 1285, 151-175 (2015).

62. Noy, T. et al. Central role of pyruvate kinase in carbon cocatabolism of Mycobacterium tuberculosis. J. Biol. Chem. 291, 7060-7069 (2016).

63. Paglia, G. et al. Monitoring metabolites consumption and secretion in cultured cells using ultra-performance liquid chromatography quadrupole-time of flight mass spectrometry (UPLC-Q-ToF-MS). Anal. Bioanal. Chem. 402, 1183-1198 (2012).

64. Benjak, A., Sala, C. \& Hartkoorn, R. C. Whole-transcriptome sequencing for high-resolution transcriptomic analysis in Mycobacterium tuberculosis. Methods Mol. Biol. 1285, 17-30 (2015).

65. Council, N. R. Guide for the Care and Use of Laboratory Animals: Eighth Edition, (The National Academies Press, Washington, DC, 2011).

\section{Acknowledgements}

This study was financially supported by NIH funding AI119573 and AI139465 for M.B., D.R.S., and E.H. and T32-GM007288 and AI138483 for E.H. S.J. was supported by NIH grant T32 AI007501. G.C. was supported by Marsden Fund NZ. J.M.T. was supported by effect:hope (Leprosy Mission Canada) on behalf of the Research to Stop Neglected Tropical Disease Transmission (R2STOP) Initiative. W.R.J. was supported by AI026170. We thank Dirk Schnappinger for providing the plasmids for making the DUC constructs and Sabine Ehrt for helping out with doxy chow. We thank John Kim, Mei Chen, Annie Zhi Dai, Bing Chen, Robert Dubin, Xusheng Zhang, and Fabien Delahaye for technical support and Kevin Pethe for critical reading of the manuscript.

\section{Author contributions}

E.J.H., G.M.C., W.R.J. and M.B. designed the research. E.J.H., D.R.S., L.B.M., S.J., J.M.T., T.F. and M.B. performed research. E.J.H. and M.B. analyzed data. E.J.H., G.M.C. and M.B. wrote the paper. 


\section{Additional information}

Supplementary Information accompanies this paper at https://doi.org/10.1038/s41467019-12224-3.

Competing interests: The authors declare no competing interests.

Reprints and permission information is available online at http://npg.nature.com/ reprintsandpermissions/

Peer review information Nature Communications thanks the anonymous reviewers for their contribution to the peer review of this work. Peer reviewer reports are available.

Publisher's note Springer Nature remains neutral with regard to jurisdictional claims in published maps and institutional affiliations. (c) Open Access This article is licensed under a Creative Commons Attribution 4.0 International License, which permits use, sharing, adaptation, distribution and reproduction in any medium or format, as long as you give appropriate credit to the original author(s) and the source, provide a link to the Creative Commons license, and indicate if changes were made. The images or other third party material in this article are included in the article's Creative Commons license, unless indicated otherwise in a credit line to the material. If material is not included in the article's Creative Commons license and your intended use is not permitted by statutory regulation or exceeds the permitted use, you will need to obtain permission directly from the copyright holder. To view a copy of this license, visit http://creativecommons.org/ licenses/by/4.0/.

(C) The Author(s) 2019 